\title{
Subject Index
}

Adenoviral vectors, hepatitis $\mathrm{B}$ virus genome transfer into mice 35-37

Antibody neutralization

human hepatocyte hepatitis B virus model 128

Tupaia belangeri primary hepatocyte model 99-101

\section{Chimpanzee}

hepatitis B virus infection, T-cell

response

human similarities 68

prospects for study 78

successful immune response 68,69

T helper cells in viral clearance 69,71

viral clearance without destruction of infected cells 68

hepatitis $\mathrm{C}$ virus infection

acute course 72,73

T-cell response

prospects for study 78

protective immunity 77

successful immune response 73-75

viral persistence mechanisms $75-77$

CPD (carboxypeptidase; gp180), duck hepatitis B virus receptor 60

Duck hepatitis B virus (DHBC)

avian hepadnaviruses $42,43,57$

cell specificity 43,44

genome 44

hepatocyte infection 57 host cell tropism

infectious entry 59-62

intracellular trafficking 63,64

immune response $48-51$

liver damage 48, 49

persistent infection after transient infection resolution 51

PreS/S protein G133E cytopathic mutant 47,48

replication 44,45

species specificity determinants $57-59$

transmission 45,46

treatment studies

DNA vaccines 52

entecavir 52,53

virion structure 44

virulence determinants 46

$\mathrm{X}$ protein knockout strains 46,47

Entecavir, duck hepatitis B virus treatment studies 52,53

Gene therapy, interferons in the woodchuck hepatitis virus model $15,16,19$

$\mathrm{Gr}-1+$ cells, hepatitis B virus mouse response 28,29

Hepatitis B virus (HBV) avian hepadnaviruses 42, 43, 57 cellular immune response 25 duck model, see Duck hepatitis B virus epidemiology 42, 56, 138 
Hepatitis B virus (HBV) (continued)

$\beta_{2}$-glycoprotein interactions 103

hepatitis incidence 25

hepatocyte infection models, see

Hepatocyte infection models

replication 33,34

species specificity $45,119,120$

transgenic mouse models, see also

uPA/RAG-2 mouse

adenoviral vectors $35-37$

advantages $34-37,39$

closed circular DNA synthesis 27,36

construction $26,35,36,38,39$

hydrodynamic injection of DNA 38, 39

immune response

chemokine receptors 28

cytokines 26,27

cytotoxic T-lymphocytes 26,27

Gr- $1+$ cells 28, 29

matrix metalloproteinases 29

lymphocyte-deficient mice 37, 38, 136

replication 36

transmission 46

treatment resistance 146

tree shrew model, see Tupaia belangeri

trimera model, see Trimera mouse model

uPA/RAG-2 mouse model, see uPA/RAG-2 mouse

vaccination 147

virion structure 33

woodchuck hepatitis virus comparison

1,37

Hepatitis $\mathrm{C}$ virus (HCV)

chimpanzee model, see Chimpanzee

classification 81, 106, 107

clinical features of infection 106

genome 81,106

overview of models

animal models 109,110

in vitro models 108

table 107

Tupaia belangeri hepatocyte infection

110-114

replication 81, 82, 106, 107

replicons, see Replicons, hepatitis $\mathrm{C}$ virus

treatment resistance 146

trimera model, see Trimera mouse model
Tupaia belangeri model, see Tupaia belangeri

uPA/RAG-2 mouse model, see uPA/RAG-2 mouse

vaccination 147

Hepatocyte infection models

duck hepatocytes crane hepatitis B virus infection

58,59

duck hepatitis B virus infection 57

hepatocyte cryopreservation for

uPA/RAG-2 mouse 141, 142

human hepatocyte hepatitis $\mathrm{B}$ virus

model

antibody neutralization studies 128

antiviral agent evaluation 130

attachment and receptor studies

127,128

cell isolation 120,121

closed circular DNA detection 123, 124

culture of primary hepatocytes

121,122

cytokine antiviral effect studies 128,129

donor characteristics 120,121

immune effector cell interactions with hepatocytes 129,130

infection

conditions 122

standardization $125-127$

limitations 56, 57, 120, 131

prospects $130-132$

viral antigen detection 123-125

Tupaia belangeri primary hepatocyte models

hepatitis B virus infection advantages and prospects 103,104

antigen secretion 99

binding and inhibition by serum 96, 97, 103

closed circular DNA formation 97, 98

monoclonal antibody neutralization studies 99-101

time-course analysis 98,99

woolly monkey hepatitis B virus infection 101-103 


\author{
hepatitis $\mathrm{C}$ virus infection \\ infectivity 111, 112 \\ prospects 114 \\ receptor studies $112-114$ \\ replication levels 112 \\ RNA detection 110,111 \\ uPA/RAG-2 mouse model, see \\ uPA/RAG-2 mouse
}

Human immunodeficiency virus

(HIV), intracellular trafficking

63,64

Interferons

antiviral activity 18, 146

gene therapy studies, woodchuck

hepatitis virus model 15, 16, 19

Liver transplantation, adoptive immunotransfer in the woodchuck

hepatitis virus model $16-18$

Major histocompatibility complex (MHC), woodchuck immune system

class I genes 7

class II genes 7

Neutralization studies, see Antibody neutralization

PreS/S protein, G133E cytopathic mutant 47, 48

Primary hepatocytes, see Hepatocyte infection models

Replicons, hepatitis C virus advantages 82

cell clone applications $88-90$

isolates for use

Con1 85, 86

genotype $1 \mathrm{~b}$ replicons 86,87

limitations 91

permissive cell lines 87,88

principles 82,83

prospects 90,91

replication efficiency determinants,

Con1 isolate 83-85

transient replication assays 89,90
Serum, hepatitis B virus infection inhibition in Tupaia belangeri

hepatocytes 96, 97, 103

T-cell

hepatitis B virus studies

chimpanzee

human similarities 68

prospects for study 78

successful immune response 68,69

T helper cells, viral clearance 69,71

viral clearance without destruction

of infected cells 68

human response 67,68

transgenic mouse model response 26,27

hepatitis $\mathrm{C}$ virus studies

chimpanzee

prospects for study 78

protective immunity 77

successful immune response $73-75$

viral persistence mechanisms $75-77$

humans 72

human study limitations 66

interactions with infected hepatocytes

129,130

trimera mouse model, hepatitis virus and

vaccine responses $152-155$

woodchuck immune system

surface markers 5,6

woodchuck hepatitis virus immune

response

chronic carriers and tolerance $12-14$

epitope mapping $10-12$

incubation period and acute phase of infection 9,10

proliferation assay 8,9

Tree shrew, see Tupaia belangeri

Trimera mouse model

advantages compared with other hepatitis

animal models 156,157

cell-mediated immune response 152-155

hepatitis B virus replication 151, 152

hepatitis $C$ virus replication 150,151

human peripheral blood mononuclear

cell transfer 148-150

humoral immune response 152 
Trimera mouse model (continued)

principles $148-150$

vaccine studies 154,155

Tupaia belangeri

animal features 96

hepatitis $\mathrm{C}$ virus infection 109,110

primary hepatocyte infection models hepatitis B virus advantages and prospects 103,104

antigen secretion 99

binding and inhibition by serum 96 , 97, 103

closed circular DNA formation 97, 98

monoclonal antibody neutralization studies 99-101

time-course analysis 98, 99

woolly monkey hepatitis B virus infection 101-103

hepatitis $\mathrm{C}$ virus

infectivity 111,112

prospects 114

receptor studies $112-114$

replication levels 112

RNA detection 110,111

uPA/RAG-2 mouse model, see

uPA/RAG-2 mouse

uPA/RAG-2 mouse

hepatocyte cryopreservation 141, 142

primary human hepatocyte transplantation hepatitis B virus infection human hepatocyte survival 138, 139

Tupaia hepatocytes 139-141

hepatitis $C$ virus infection 142,143

rationale 135,136

technique 136,137

principles 136

Woodchuck hepatitis virus (WHV)

advantages of woodchuck model 1,2

discovery 1

endemics 1

hepatitis B virus comparison 1, 37

prospects for woodchuck model 19

T-cell immune response

chronic carriers and tolerance 12-14

epitope mapping $10-12$

incubation period and acute phase of infection 9, 10

proliferation assay 8,9

treatment of chronic infection adoptive immunotransfer by liver transplantation 16-18

interferon gene therapy $15,16,19$

nucleoside analogs 14

woodchuck immune system

cytokines 3-5

major histocompatibility complex class I genes 7

class II genes 7

T-cell surface markers 5, 6

Woolly monkey hepatitis B virus, Tupaia

belangeri hepatocyte infection 101-103 